\title{
m-POLAR FUZZY HYPERIDEALS IN LA-SEMIHYPERGROUPS
}

\author{
AHMED ELMOASRY ${ }^{1,2, *}$, NAVEED YAQOOB $^{1}$ \\ ${ }^{1}$ Department of Mathematics, College of Science Al-Zulfi, Majmaah University, Al-Zulfi, Saudi Arabia \\ ${ }^{2}$ Department of Mathematics, Faculty Of science, Aswan University, Aswan, Egypt \\ ${ }^{*}$ Corresponding author: a.elmoasry@mu.edu.sa
}

\begin{abstract}
In this paper, we initiate a study of $m$-polar fuzzy sets in hyperstructure theory, particularly in left almost semihypergroups. We define an $m$-polar fuzzy left (right, two sided) hyperideal in a left almost semihypergroup and provided some results on these $m$-polar fuzzy left (right, two sided) hyperideals.
\end{abstract}

\section{Introduction}

In 1934, hyperstructure theory was introduced by a French mathematician Marty [1] and then, several authors continued their researches in this direction. Hyperstructures are now widely studied from theoretical point of view and for their applications in many subjects of pure and applied mathematics. Some preliminary results and definitions about hyperstructure theory can be found in the books written by Corsini [2] and Vougiouklis [3]. The concept of a semihypergroup is a generalization of the concept of a semigroup. Many authors studied different aspects of semihypergroups. Some principal notions about semihypergroup theory can be found in [4]. In 2011, Hila and Dine [5] introduced the concept of non-associative semihypergroups (LA-semihypergroups) which is a generalization of semigroups, semihypergroups, and LA-semigroups. Later, Yaqoob et al. [6] explored this concept further and studied intra-regular left almost semihypergroups by their hyperideals using pure left identity. Other results on LA-semihypergroups can be found in [7-9].

Received 2019-03-07; accepted 2019-04-01; published 2019-05-01.

1991 Mathematics Subject Classification. 20N20, 03E72.

Key words and phrases. LA-semihypergroups; hyperideals; $m$-polar fuzzy sets; $m$-polar fuzzy hyperideals.

(C)2019 Authors retain the copyrights of their papers, and all open access articles are distributed under the terms of the Creative Commons Attribution License. 
In 1965, Zadeh [10] introduced the notion of a fuzzy subset of a non-empty set $X$, as a function from $X$ to $[0,1]$. After the introduction of fuzzy sets, several researchers conducted the researches on generalizations of fuzzy sets with huge applications in computer, logics, automata and many branches of pure and applied mathematics. In 1971, Rosenfeld [11] defined the concept of a fuzzy subgroup of a group, and provided a tool and a novel approach to establish the fuzzy analogs of many results in group theory. However, in 1979, Anthony and Sherwood [12] generalized the Rosenfeld's definition of fuzzy group. Sherwood [13] defined products of fuzzy subgroups using $t$-norms and gave some properties of these products. Asaad [14] proposed the idea about the relation between structure of a group and the structure of a fuzzy subgroup. Mukherjee and Bhattacharya, introduced the concept of a fuzzy normal subgroup and a fuzzy coset in [15]. In 1981, Das [16] introduced the concept of level subsets and level subgroups. Fuzzy set theory has been developed in the context of hyperalgebraic structure theory. In [17], Davvaz introduced the concept of fuzzy hyperideals in a semihypergroup. More on fuzzy hyperstructures one can find in [18].

In 1994, Zhang [19] generalized the idea of a fuzzy set and gave the concept of a bipolar fuzzy set on a given set $X$ as a map, which associates each element of $X$, to a real number, in the interval $[-1,1]$. In 2014, Chen et al. [20] introduced the idea of $m$-polar fuzzy sets, as an extension of bipolar fuzzy sets and showed that bipolar fuzzy sets and 2-polar fuzzy sets are cryptomorphic mathematical notions and that we can obtain concisely one from the corresponding one [20]. Recently, Akram with others applied the concept of $m$-polar fuzzy sets in different directions, like, Lie algebras [21, 22], graph theory [23, 24], groups [25], lattices [26] and matroids [27]. Akrem published a book on $m$-polar fuzzy graphs [28], in which he provided several types of $m$-polar fuzzy graphs with applications. Al-Masarwah and Ahmad [29] studied $m$-polar $(\alpha, \beta)$-fuzzy ideals in BCK/BCI-Algebra.

\section{Preliminaries}

Definition 2.1. A map * $H \times H \rightarrow \mathcal{P}^{*}(H)$ is called a hyperoperation or a join operation on a set $H$, where $H$ is a non-empty set and $\mathcal{P}^{*}(H)=\mathcal{P}(H) \backslash\{\emptyset\}$ denotes the set of all non-empty subsets of $H$.

Definition 2.2. A hypergroupoid is a pair $(H, *)$, where $*$ is a hyperoperation on $H$.

Definition 2.3. $[5,6]$ A hypergroupoid $(H, *)$ is called an LA-semihypergroup if for all $x, y, z \in H$,

$$
(x * y) * z=(z * y) * x .
$$

The law $(x * y) * z=(z * y) * x$ is called a left invertive law. In an LA-semihypergroup, the medial law $(x * y) *(z * w)=(x * z) *(y * w)$ holds for all $x, y, z, w \in H$.

Let $A$ and $B$ be two non-empty subsets of $H$. Then we define 


$$
A * B=\bigcup_{a \in A, b \in B} a * b, \quad a * A=\{a\} * A \text { and } a * B=\{a\} * B .
$$

Definition 2.4. An element $e \in H$ is called a left identity (resp., pure left identity) if for all $x \in H, x \in e * x$ $($ resp., $x=e * x)$.

An LA-semihypergroup may or may not contains a left identity and a pure left identity.

Lemma 2.1. [6] Let $H$ be an LA-semihypergroup with a pure left identity $e$. Then $x *(y * z)=y *(x * z)$ holds for all $x, y, z \in H$.

Lemma 2.2. [6] Let $H$ be an LA-semihypergroup with a pure left identity e. Then the paramedial law $(x * y) *(z * w)=(w * z) *(y * x)$ holds for all $x, y, z, w \in H$.

Definition 2.5. [5] Let $H$ be an LA-semihypergroup. A non-empty subset $A$ of $H$ is called a sub LAsemihypergroup of $H$ if $x * y \subseteq A$ for every $x, y \in A$.

Definition 2.6. [5] A subset I of an LA-semihypergroup $H$ is called a right (resp., left) hyperideal of $H$ if $I * H \subseteq I$ (resp., $H * I \subseteq I$ ) and is called a hyperideal if it is two-sided hyperideal.

Definition 2.7. [6] By a bi-hyperideal (resp., generalized bi-hyperideal) of an LA-semihypergroup H, we mean a sub LA-semihypergroup (resp., non-empty subset) $B$ of $H$ such that $(B * H) * B \subseteq B$.

Definition 2.8. [6] By an interior hyperideal of an LA-semihypergroup $H$, we mean a subset $A$ of $H$ such that $(H * A) * H \subseteq A$.

Definition 2.9. An LA-semihypergroup $H$ is called a regular LA-semihypergroup if for every $x \in H, x \in$ $(x * y) * x$, for some $y \in H$.

An element $a$ of $H$ is called a left regular element of $H$ if there exists $x \in H$ such that $a \in x *(a * a)$ and $H$ is called left regular if every element of $H$ is left regular.

Definition 2.10. [20] An m-polar fuzzy set (or a $[0,1]^{m}$-set) on $X$ is a mapping $\widehat{M}: X \rightarrow[0,1]^{m}$. The membership value of every element $x \in X$ is denoted by

$$
\widehat{M}(x)=\left(p_{1} \circ \widehat{M}(x), p_{2} \circ \widehat{M}(x), \ldots, p_{m} \circ \widehat{M}(x)\right)
$$

where $p_{i}:[0,1]^{m} \rightarrow[0,1]$ is the $i$-th projection mapping $(i \in m)$.

Note that $[0,1]^{m}$ ( $m$-power of $\left.[0,1]\right)$ is considered a poset with the point-wise order $\preceq$, where $m$ is an arbitrary ordinal number (we make an appointment that $m=\{n \mid n<m\}$ when $m>0$ ), $\preceq$ is defined by $u \preceq v \Leftrightarrow p_{i}(u) \preceq p_{i}(v)$ for each $i \in m\left(u, v \in[0,1]^{m}\right)$. Also, $\widehat{0}=(0,0, \cdots, 0)$ is the smallest element in $[0,1]^{m}$ and $\widehat{1}=(1,1, \cdots, 1)$ is the largest element in $[0,1]^{m}$. 


\section{3. $m$-POLAR FUZZY HYPERIDEALS}

In this section, we will provide some results on $m$-polar fuzzy hyperideals. Let $\widehat{M}_{1}$ and $\widehat{M}_{2}$ be two $m$-polar fuzzy sets in an LA-semihypergroup $H$. Then for any $x \in H$, we define the following:

$$
\begin{aligned}
& \left(\widehat{M}_{1} \cap \widehat{M}_{2}\right)(x)=\min \left\{\widehat{M}_{1}(x), \widehat{M}_{2}(x)\right\} \\
& \left(\widehat{M}_{1} \cup \widehat{M}_{2}\right)(x)=\max \left\{\widehat{M}_{1}(x), \widehat{M}_{2}(x)\right\}
\end{aligned}
$$

and

$$
\left(\widehat{M}_{1} \odot \widehat{M}_{2}\right)(x)= \begin{cases}\sup _{x \in y * z}\left\{\min \left\{\widehat{M}_{1}(y), \widehat{M}_{2}(z)\right\}\right. & \text { if } x \in y * z \\ (0,0, \ldots, 0) & \text { if } x \notin y * z .\end{cases}
$$

We denote the set of all $m$-polar fuzzy subsets in an LA-semihypergroup $H$ by $F_{m}(H)$. For any $\widehat{M}_{1}$, $\widehat{M}_{2} \in F_{m}(H)$,

$$
\widehat{M}_{1} \subseteq \widehat{M}_{2} \Leftrightarrow \widehat{M}_{1}(x) \preceq \widehat{M}_{2}(x),
$$

for each $x \in H$.

Proposition 3.1. Let $H$ be an LA-semihypergroup. Then the set $\left(F_{m}(H), \odot\right)$ is an LA-semihypergroup.

Proof. The proof is straightforward.

Proposition 3.2. If $H$ is an LA-semihypergroup, then the medial law

$$
\left(\widehat{M}_{1} \odot \widehat{M}_{2}\right) \odot\left(\widehat{M}_{3} \odot \widehat{M}_{4}\right)=\left(\widehat{M}_{1} \odot \widehat{M}_{3}\right) \odot\left(\widehat{M}_{2} \odot \widehat{M}_{4}\right)
$$

holds in $F_{m}(H)$, for all $\widehat{M}_{1}, \widehat{M}_{2}, \widehat{M}_{3}$ and $\widehat{M}_{4}$ in $F_{m}(H)$.

Proof. The proof is straightforward.

Proposition 3.3. Let $H$ be an LA-semihypergroup with a pure left identity. Then the following properties hold in $F_{m}(H)$ :

(i): $\widehat{M}_{1} \odot\left(\widehat{M}_{2} \odot \widehat{M}_{3}\right)=\widehat{M}_{2} \odot\left(\widehat{M}_{1} \odot \widehat{M}_{3}\right)$;

(ii): $\left(\widehat{M}_{1} \odot \widehat{M}_{2}\right) \odot\left(\widehat{M}_{3} \odot \widehat{M}_{4}\right)=\left(\widehat{M}_{4} \odot \widehat{M}_{3}\right) \odot\left(\widehat{M}_{2} \odot \widehat{M}_{1}\right)$;

for all $\widehat{M}_{1}, \widehat{M}_{2}, \widehat{M}_{3}$ and $\widehat{M}_{4}$ in $F_{m}(H)$. 
Proof. (i) Let $\widehat{M}_{1}, \widehat{M}_{2}$ and $\widehat{M}_{3}$ be in $F_{m}(H)$. There exist $x$ and $y$ in $H$ such that $z \in x * y$. Then

$$
\begin{aligned}
\left(\widehat{M}_{1} \odot\left(\widehat{M}_{2} \odot \widehat{M}_{3}\right)\right)(z) & =\sup _{z \in x * y}\left\{\min \left\{\widehat{M}_{1}(x),\left(\widehat{M}_{2} \odot \widehat{M}_{3}\right)(y)\right\}\right\} \\
& =\sup _{z \in x * y}\left\{\min \left\{\widehat{M}_{1}(x), \sup _{y \in t * w}\left\{\min \left\{\widehat{M}_{2}(t), \widehat{M}_{3}(w)\right\}\right\}\right\}\right\} \\
& =\sup _{z \in x * y} \sup _{y \in t * w}\left\{\min \left\{\widehat{M}_{1}(x), \widehat{M}_{2}(t), \widehat{M}_{3}(w)\right\}\right\} \\
& =\sup _{z \in x *(t * w)}\left\{\min \left\{\widehat{M}_{1}(x), \widehat{M}_{2}(t), \widehat{M}_{3}(w)\right\}\right\} \\
& =\sup _{z \in t *(x * w)}\left\{\min \left\{\widehat{M}_{2}(t), \widehat{M}_{1}(x), \widehat{M}_{3}(w)\right\}\right\}, \\
& =\sup _{z \in t * m} \sup \left\{\min \left\{\widehat{M}_{2}(t), \widehat{M}_{1}(x), \widehat{M}_{3}(w)\right\}\right\} \\
& =\sup _{z \in t * m}\left\{\min \left\{\widehat{M}_{2}(t), \sup _{m \in x * w}\left\{\min \left\{\widehat{M}_{1}(x), \widehat{M}_{3}(w)\right\}\right\}\right\}\right\} \\
& =\sup _{z \in t * m}\left\{\min \left\{\widehat{M}_{2}(t),\left(\widehat{M}_{1} \odot \widehat{M}_{3}\right)(m)\right\}\right\} \\
& =\left(\widehat{M}_{2} \odot\left(\widehat{M}_{1} \odot \widehat{M}_{3}\right)\right)(z) .
\end{aligned}
$$

Let $z$ be an element of $H$ such that $z \notin x * y$, for some $x, y \in H$. Then we have

$$
\left(\widehat{M}_{1} \odot\left(\widehat{M}_{2} \odot \widehat{M}_{3}\right)\right)(z)=(0,0, \ldots, 0)=\left(\widehat{M}_{2} \odot\left(\widehat{M}_{1} \odot \widehat{M}_{3}\right)\right)(z) .
$$

Hence this shows that $\widehat{M}_{1} \odot\left(\widehat{M}_{2} \odot \widehat{M}_{3}\right)=\widehat{M}_{2} \odot\left(\widehat{M}_{1} \odot \widehat{M}_{3}\right)$ holds in $F_{m}(H)$.

(2) The proof is similar to (1).

Lemma 3.1. Let $H$ be an LA-semihypergroup with a pure left identity. Then for all $x \in H, \mathcal{H} \odot \mathcal{H}=\mathcal{H}$, where $\mathcal{H}(x)=(1,1, \ldots, 1)$.

Proof. The proof is straightforward.

Definition 3.1. An m-polar fuzzy set $\widehat{M}$ of an LA-semihypergroup $H$ is called an m-polar fuzzy sub LAsemihypergroup of $H$ if for all $x, y \in H$, the following condition hold:

$$
\inf _{z \in x * y}\{\widehat{M}(z)\} \succeq \min \{\widehat{M}(x), \widehat{M}(y)\}
$$

That is

$$
\inf _{z \in x * y}\left\{p_{i} \circ \widehat{M}(z)\right\} \succeq \min \left\{p_{i} \circ \widehat{M}(x), p_{i} \circ \widehat{M}(y)\right\}
$$

for each $i=1,2,3, \cdots, m$. 
Example 3.1. Consider an LA-semihypergroup $H=\{e, a, b, c, d\}$, with the hyperoperation " * " defined as follow:

\begin{tabular}{c|ccccc}
$*$ & $e$ & $a$ & $b$ & $c$ & $d$ \\
\hline$e$ & $e$ & $e$ & $e$ & $e$ & $e$ \\
$a$ & $e$ & $a$ & $a$ & $a$ & $a$ \\
$b$ & $e$ & $a$ & $a$ & $\{a, d\}$ & $a$ \\
$c$ & $e$ & $a$ & $\{a, b\}$ & $\{a, c\}$ & $\{a, d\}$ \\
$d$ & $e$ & $a$ & $a$ & $\{a, b\}$ & $a$
\end{tabular}

We define a 5-polar fuzzy set $\widehat{M}: H \rightarrow[0,1]^{5}$ as follows:

$$
\widehat{M}(x)= \begin{cases}(0.6,0.7,0.8,0.8,0.9) & \text { if } x=e \\ (0.5,0.6,0.8,0.8,0.8) & \text { if } x=a \\ (0.4,0.4,0.7,0.7,0.7) & \text { if } x \in\{b, d\} \\ (0.1,0.3,0.2,0.4,0.1) & \text { if } x=c .\end{cases}
$$

By routine calculations, it is easy to verify that $\widehat{M}$ is a 5-polar fuzzy sub LA-semihypergroup of $H$.

Definition 3.2. An m-polar fuzzy set $\widehat{M}$ of an LA-semihypergroup $H$ is called an m-polar fuzzy left (resp., right) hyperideal of $H$ if for all $x, y \in H$, the following condition hold:

$$
\left.\inf _{z \in x * y}\{\widehat{M}(z)\} \succeq \widehat{M}(y) \text { (resp., } \inf _{z \in x * y}\{\widehat{M}(z)\} \succeq \widehat{M}(x)\right)
$$

That is

$$
\left.\inf _{z \in x * y}\left\{p_{i} \circ \widehat{M}(z)\right\} \succeq p_{i} \circ \widehat{M}(y) \text { (resp., } \inf _{z \in x * y}\left\{p_{i} \circ \widehat{M}(z)\right\} \succeq p_{i} \circ \widehat{M}(x)\right)
$$

for each $i=1,2,3, \cdots, m$.

Definition 3.3. An m-polar fuzzy set $\widehat{M}$ of an LA-semihypergroup $H$ is called an m-polar fuzzy hyperideal of $H$ if for all $x, y \in H$, the following condition hold:

$$
\inf _{z \in x * y}\{\widehat{M}(z)\} \succeq \max \{\widehat{M}(x), \widehat{M}(y)\}
$$

That is

$$
\inf _{z \in x * y}\left\{p_{i} \circ \widehat{M}(z)\right\} \succeq \max \left\{p_{i} \circ \widehat{M}(x), p_{i} \circ \widehat{M}(y)\right\}
$$

for each $i=1,2,3, \cdots, m$. 
Example 3.2. Consider an LA-semihypergroup $H=\{e, a, b, c, d\}$, with the hyperoperation " * defined as follow:

\begin{tabular}{c|ccccc}
$*$ & $e$ & $a$ & $b$ & $c$ & $d$ \\
\hline$e$ & $e$ & $e$ & $e$ & $e$ & $e$ \\
$a$ & $e$ & $a$ & $\{e, b\}$ & $a$ & $a$ \\
$b$ & $e$ & $\{e, b\}$ & $b$ & $\{e, b\}$ & $\{e, b\}$ \\
$c$ & $e$ & $a$ & $\{e, b\}$ & $\{a, c, d\}$ & $d$ \\
$d$ & $e$ & $a$ & $\{e, b\}$ & $\{a, d\}$ & $\{a, d\}$
\end{tabular}

We define a 3-polar fuzzy set $\widehat{M}: H \rightarrow[0,1]^{3}$ as follows:

$$
\widehat{M}(x)= \begin{cases}(0.8,0.9,0.8) & \text { if } x=e \\ (0.5,0.5,0.5) & \text { if } x=a \\ (0.7,0.7,0.6) & \text { if } x=b \\ (0.1,0.2,0.2) & \text { if } x=c \\ (0.3,0.4,0.3) & \text { if } x=d .\end{cases}
$$

By routine calculations, it is easy to verify that $\widehat{M}$ is a 3-polar fuzzy hyperideal of $H$.

Definition 3.4. Let $H$ be an LA-semihypergroup. Let $\widehat{t}=\left(t_{1}, t_{2}, \ldots, t_{m}\right) \in[0,1]^{m}$ and $\widehat{M}$ be an m-polar fuzzy set in $H$. Then, the set

(i): $\widehat{M}_{\widehat{t}}=\{x \in H \mid \widehat{M}(x) \succeq \widehat{t}\}$ is called a $\widehat{t}$-level cut of $\widehat{M}$.

(ii): $\widehat{M}_{\widehat{t}}^{s}=\{x \in H \mid \widehat{M}(x) \succ \widehat{t}\}$ is called a strong $\widehat{t}$-level cut of $\widehat{M}$.

(iii): $\operatorname{Im}(\widehat{M})$ is called the image of $\widehat{M}$.

Theorem 3.1. Let $\widehat{M}$ be an m-polar fuzzy set in $H$, such that the least upper bound $\widehat{t}_{0}$ of $\operatorname{Im}(\widehat{M})$ exist. Then the following conditions are equivalent:

(i): $\widehat{M}$ is an m-polar fuzzy sub LA-semihypergroup of $H$.

(ii): For all $\widehat{t} \in \operatorname{Im}(\widehat{M})$, the non-empty $\widehat{t}$-level subset $\widehat{M}_{\widehat{t}}$ of $\widehat{M}$ is a sub LA-semihypergroup of $H$.

(iii): For all $\widehat{t} \in \operatorname{Im}(\widehat{M}) \backslash \widehat{t}_{0}$, the non-empty strong $\widehat{t}$-level subset $\widehat{M}_{\hat{t}}^{s}$ of $\widehat{M}$ is a sub LA-semihypergroup of $H$.

(iv): For all $\widehat{t} \in[0,1]^{m}$, the non-empty strong $\widehat{t}$-level subset $\widehat{M}_{\widehat{t}}^{s}$ of $\widehat{M}$ is a sub LA-semihypergroup of $H$.

(v): For all $\widehat{t} \in[0,1]^{m}$, the non-empty $\widehat{t}$-level subset $\widehat{M}_{\widehat{t}}$ of $\widehat{M}$ is a sub LA-semihypergroup of $H$.

Proof. $(i) \rightarrow(i v)$. Let $\widehat{M}$ be a $m$-polar fuzzy sub LA-semihypergroup of $H, \widehat{t} \in[0,1]^{m}$, and $x, y \in \widehat{M}_{\widehat{t}}^{s}$. Then we have $\widehat{M}(x), \widehat{M}(y) \succ \widehat{t}$. Thus, $\min \{\widehat{M}(x), \widehat{M}(y)\} \succ \widehat{t}$. Since $\widehat{M}$ is a sub LA-semihypergroup of $H$, so $\inf _{z \in x * y} \widehat{M}(z) \succ \widehat{t}$. Thus $z \in \widehat{M}_{\widehat{t}}^{s}$ for each $z \in x * y$. Hence $\widehat{M}_{\widehat{t}}^{s}$ is a sub LA-semihypergroup of $H$. 
$($ iv $) \rightarrow($ iii $)$. It is clear.

$($ iii $) \rightarrow(i i)$. Let $\widehat{t} \in \operatorname{Im}(\widehat{M})$. Then $\widehat{M}_{\widehat{t}}$ is non-empty. Since $\widehat{M}_{\widehat{t}}=\bigcap_{\widehat{t} \prec \widehat{\alpha}}^{H} \widehat{M}_{\widehat{\alpha}}$, where $\widehat{\alpha} \in \operatorname{Im}(\widehat{M}) \backslash \widehat{t}_{0}$. Then by (iii) we get that $\widehat{M}_{\widehat{t}}$ is a sub LA-semihypergroup of $H$.

$(i i) \rightarrow(v)$. Let $\widehat{t} \in[0,1]^{m}$ and $\widehat{M}_{\widehat{t}}$ be non-empty. Suppose that $x, y \in \widehat{M}_{\widehat{t}}$. Then, we have $\widehat{M}(x), \widehat{M}(y) \succeq \widehat{t}$. Let $\widehat{\alpha}=\min \{\widehat{M}(x), \widehat{M}(y)\}$. It is clear that $\widehat{\alpha} \succeq \widehat{t}$. Thus $x, y \in \widehat{M}_{\widehat{t}}$ and $\widehat{\alpha} \in \operatorname{Im}(\widehat{M})$, by (ii) $\widehat{M}_{\widehat{\alpha}}$ is a sub LA-semihypergroup of $H$, hence $z \in \widehat{M}_{\widehat{\alpha}}$ for each $z \in x * y$. Then we have $\inf _{z \in x * y} \widehat{M}(z) \succeq \widehat{\alpha} \succeq \widehat{t}$. Therefore $x * y \subseteq \widehat{M}_{\widehat{t}}$. Then $\widehat{M}_{\widehat{t}}$ is a sub LA-semihypergroup of $H$.

$(v) \rightarrow(i)$. Assume that the non-empty set $\widehat{M}_{\widehat{t}}$ is a sub LA-semihypergroup of $H$, for any $\widehat{t} \in[0,1]^{m}$. Let $x, y \in H$. Let us take $\widehat{t}=\min \{\widehat{M}(x), \widehat{M}(y)\}$. Then $\widehat{M}(x), \widehat{M}(y) \succeq \widehat{t}$. Thus $x, y \in \widehat{M}_{\widehat{t}}$. Since $\widehat{M}_{\widehat{t}}$ is a sub LA-semihypergroup of $H$, so for each $z \in x * y, z \in \widehat{M}_{\widehat{t}}$. Thus,

$$
\inf _{z \in x * y} \widehat{M}(z) \succeq \widehat{t}=\min \{\widehat{M}(x), \widehat{M}(y)\}
$$

This shows that $\widehat{M}$ is an $m$-polar fuzzy sub LA-semihypergroup of $H$. This completes the proof.

Theorem 3.2. Let $\widehat{M}$ be an m-polar fuzzy set in $H$, such that the least upper bound $\widehat{t}_{0}$ of $\operatorname{Im}(\widehat{M})$ exist. Then the following conditions are equivalent:

(i): $\widehat{M}$ is an m-polar fuzzy left (resp., right) hyperideal of $H$.

(ii): For all $\widehat{t} \in \operatorname{Im}(\widehat{M})$, the non-empty $\widehat{t}$-level subset $\widehat{M}_{\widehat{t}}$ of $\widehat{M}$ is a left (resp., right) hyperideal of $H$.

(iii): For all $\widehat{t} \in \operatorname{Im}(\widehat{M}) \backslash \widehat{t_{0}}$, the non-empty strong $\widehat{t}$-level subset $\widehat{M}_{\widehat{t}}^{s}$ of $\widehat{M}$ is a left (resp., right) hyperideal of $H$.

(iv): For all $\widehat{t} \in[0,1]^{m}$, the non-empty strong $\widehat{t}$-level subset $\widehat{M}_{\hat{t}}^{s}$ of $\widehat{M}$ is a left (resp., right) hyperideal of $H$.

(v): For all $\widehat{t} \in[0,1]^{m}$, the non-empty $\widehat{t}$-level subset $\widehat{M}_{\widehat{t}}$ of $\widehat{M}$ is a left (resp., right) hyperideal of $H$.

Proof. The proof is similar to the proof of Theorem 3.1.

Theorem 3.3. An m-polar fuzzy subset $\widehat{M}$ of an LA-semihypergroup $H$ is an m-polar fuzzy

(1) sub LA-semihypergroup of $H$ if and only if $\widehat{M} \odot \widehat{M} \subseteq \widehat{M}$,

(2) left hyperideal of $H$ if and only if $\mathcal{H} \odot \widehat{M} \subseteq \widehat{M}$,

(3) right hyperideal of $H$ if and only if $\widehat{M} \odot \mathcal{H} \subseteq \widehat{M}$,

(4) hyperideal of $H$ if and only if $\mathcal{H} \odot \widehat{M} \subseteq \widehat{M}$ and $\widehat{M} \odot \mathcal{H} \subseteq \widehat{M}$. 
Proof. (1) Let $\widehat{M}$ be an $m$-polar fuzzy sub LA-semihypergroup of $H$ and $z \in H$. Let us suppose that $z \in x * y$ for $x, y \in H$. Then

$$
\begin{aligned}
(\widehat{M} \odot \widehat{M})(z) & =\sup _{z \in x * y}\{\min \{\widehat{M}(x), \widehat{M}(y)\} \\
& \preceq \sup _{z \in x * y}\left\{\inf _{z \in x * y} \widehat{M}(z)\right\} \\
& \preceq \sup _{z \in x * y}\{\widehat{M}(x * y)\}=\widehat{M}(z) .
\end{aligned}
$$

Therefore $(\widehat{M} \odot \widehat{M}) \subseteq \widehat{M}$. If there do not exist any $x, y \in H$ such that $z \in x * y$, then

$$
(\widehat{M} \odot \widehat{M})(z)=0 \preceq \widehat{M}(z) .
$$

Hence for all cases $\widehat{M} \odot \widehat{M} \subseteq \widehat{M}$.

Conversely, let us assume that $\widehat{M} \odot \widehat{M} \subseteq \widehat{M}$ holds for all $m$-polar fuzzy subsets of $H$. Let $x, y \in H$. Then, we have

$$
\begin{aligned}
\inf _{z \in x * y} \widehat{M}(z) & \succeq \inf _{z \in x * y}\{(\widehat{M} \odot \widehat{M})(z)\} \\
& =\inf _{z \in x * y}\left\{\sup _{z \in x * y}\{\min \{\widehat{M}(x), \widehat{M}(y)\}\}\right. \\
& =\min \{\widehat{M}(x), \widehat{M}(y)\} .
\end{aligned}
$$

This means that $\widehat{M}$ is an $m$-polar fuzzy sub LA-semihypergroup of $H$. The other cases can be seen in a similar way.

Theorem 3.4. If $\left\{\widehat{M}_{i}\right\}_{i \in \Lambda}$ is a family of m-polar fuzzy left (resp., right) hyperideals of an LA-semihypergroup $H$, then $\bigcap_{i \in \Lambda} \widehat{M}_{i}$ is also a fuzzy left (resp., right) hyperideal of $H$.

Proof. Let $\left\{\widehat{M}_{i}\right\}_{i \in \Lambda}$ be a family of $m$-polar fuzzy left hyperideals of an LA-semihypergroup $H$ and let $x, y \in H$. Then we have

$$
\begin{aligned}
\bigcap_{i \in \Lambda} \widehat{M}_{i}(y) & =\bigwedge_{i \in \Lambda}\left\{\widehat{M}_{i}(y)\right\} \preceq \bigwedge_{i \in \Lambda}\left\{\inf _{z \in x * y}\left\{\widehat{M}_{i}(z)\right\}\right\} \\
& =\inf _{z \in x * y}\left\{\bigwedge_{i \in \Lambda}\left\{\widehat{M}_{i}(z)\right\}\right\} \\
& =\inf _{z \in x * y}\left\{\bigcap_{i \in \Lambda} \widehat{M}_{i}(z)\right\} .
\end{aligned}
$$

Hence $\bigcap_{i \in \Lambda} \widehat{M}_{i}$ is an $m$-polar fuzzy left hyperideal of LA-semihypergroup $H$. Similarly we can prove it for right hyperideals. This completes the proof. 
Let $H$ be an LA-semihypergroup and let $\emptyset \neq A \subseteq H$. Then the $m$-polar fuzzy characteristic function $\chi_{\widetilde{M}}^{A}$ of $A$ is defined by

$$
\chi_{\vec{M}}^{A}(x)=\left\{\begin{array}{ll}
(1,1, \ldots, 1) & \text { if } x \in A \\
(0,0, \ldots, 0) & \text { if } x \notin A
\end{array} .\right.
$$

Theorem 3.5. Let $A$ be a nonempty subset of an LA-semihypergroup H. Then A is a sub LA-semihypergroup (resp., left hyperideal, right hyperideal) of $H$ if and only if $\chi_{\vec{M}}^{A}$ is an m-polar fuzzy sub LA-semihypergroup (resp., left hyperideal, right hyperideal) of $H$.

Proof. Let $A$ be a left hyperideal of $H$. For any $x, y \in H$, we have the following cases:

Case (1) : If $x, y \in A$, then $x * y \subseteq A$. Then $\inf _{z \in x * y} \chi_{\widehat{M}}^{A}(z)=\widehat{1}$ and $\chi_{\widehat{M}}^{A}(y)=\widehat{1}$. Therefore

$$
\inf _{z \in x * y} \chi_{\bar{M}}^{A}(z)=\chi_{\bar{M}}^{A}(y)
$$

Case (2) : If $x, y \notin A$, then $\chi_{\widehat{M}}^{A}(y)=\widehat{0}$. So

$$
\inf _{z \in x * y} \chi_{\widehat{M}}^{A}(z) \succeq \chi_{\widehat{M}}^{A}(y)
$$

Case (3): If $x \in A$ and $y \notin A$, then $\chi_{\widehat{M}}^{A}(y)=\widehat{0}$. So

$$
\inf _{z \in x * y} \chi_{\bar{M}}^{A}(z) \succeq \chi_{\bar{M}}^{A}(y)
$$

Case (4): If $x \notin A$ and $y \in A$, then $x * y \subseteq A$. Then $\inf _{z \in x * y} \chi_{\widehat{M}}^{A}(z)=\widehat{1}$ and $\chi_{\widehat{M}}^{A}(y)=\widehat{1}$. Therefore

$$
\inf _{z \in x * y} \chi_{\widehat{M}}^{A}(z)=\chi_{\widehat{M}}^{A}(y) .
$$

Hence $\chi_{\vec{M}}^{A}$ is an $m$-polar fuzzy left hyperideal of $H$.

Conversely, suppose $\chi_{\widehat{M}}^{A}$ is an $m$-polar fuzzy left hyperideal of $H$ and let $x \in H$ and $y \in A$. Then we have

$$
\inf _{z \in x * y} \chi_{\widehat{M}}^{A}(z) \succeq \chi_{\widehat{M}}^{A}(y)=\widehat{1}
$$

But, we know that $\inf _{z \in x * y} \chi_{\widehat{M}}^{A}(z) \preceq \widehat{1}$. Therefore $\inf _{z \in x * y} \chi_{\widehat{M}}^{A}(z)=\widehat{1}$. This implies that $z \in A$ for each $z \in x * y$. Hence $A$ is a left hyperideal of $H$. The other cases can be seen in a similar way.

Proposition 3.4. Let $\widehat{M}_{1}$ be an m-polar fuzzy right hyperideal of $H$ and $\widehat{M}_{2}$ be an m-polar fuzzy left hyperideal of $H$. Then $\widehat{M}_{1} \odot \widehat{M}_{2} \subseteq \widehat{M}_{1} \cap \widehat{M}_{2}$. 
Proof. Let $\widehat{M}_{1}$ be an $m$-polar fuzzy right hyperideal of $H$ and $\widehat{M}_{2}$ be an $m$-polar fuzzy left hyperideal of $H$. Let $z \in H$ and suppose that there exist $x, y \in H$ such that $z \in x * y$. Then

$$
\begin{aligned}
\left(\widehat{M}_{1} \odot \widehat{M}_{2}\right)(z) & =\sup _{z \in x * y}\left\{\min \left\{\widehat{M}_{1}(x), \widehat{M}_{2}(y)\right\}\right\} \\
& \preceq \sup _{z \in x * y}\left\{\min \left\{\inf _{z \in x * y}\left\{\widehat{M}_{1}(z)\right\}, \inf _{z \in x * y}\left\{\widehat{M}_{2}(z)\right\}\right\}\right\} \\
& =\min \left\{\widehat{M}_{1}(z), \widehat{M}_{2}(z)\right\} \\
& =\left(\widehat{M}_{1} \cap \widehat{M}_{2}\right)(z) .
\end{aligned}
$$

Let us suppose that there do not exist $x, y \in H$ such that $z \in x * y$. Then, $\left(\widehat{M}_{1} \odot \widehat{M}_{2}\right)(z)=\widehat{0} \preceq\left(\widehat{M}_{1} \cap \widehat{M}_{2}\right)(z)$. This completes the proof.

Lemma 3.2. Let $H$ be an LA-semihypergroup with a pure left identity. Then every m-polar fuzzy right hyperideal is an m-polar fuzzy hyperideal.

Proof. The proof is straightforward.

Theorem 3.6. If $\widehat{M}$ is an m-polar fuzzy left hyperideal of $H$ with a pure left identity, then $\widehat{M} \cup(\widehat{M} \odot \mathcal{H})$ and $\widehat{M} \cup(\widehat{M} \odot \widehat{M})$ are m-polar fuzzy hyperideals of $H$.

Proof. Let $\widehat{M}$ be an $m$-polar fuzzy left hyperideal of $H$. We have

$$
\begin{aligned}
(\widehat{M} \cup(\widehat{M} \odot \mathcal{H})) \odot \mathcal{H} & =(\widehat{M} \odot \mathcal{H}) \cup((\widehat{M} \odot \mathcal{H}) \odot \mathcal{H}) \\
& =(\widehat{M} \odot \mathcal{H}) \cup((\mathcal{H} \odot \mathcal{H}) \odot \widehat{M}) \\
& =(\widehat{M} \odot \mathcal{H}) \cup(\mathcal{H} \odot \widehat{M})(\text { by Lemma 3.1) } \\
& \subseteq(\widehat{M} \odot \mathcal{H}) \cup \widehat{M} .
\end{aligned}
$$

Hence, $\widehat{M} \cup(\widehat{M} \odot \mathcal{H})$ is an $m$-polar fuzzy right hyperideal of $H$. By Lemma 3.2, $\widehat{M} \cup(\widehat{M} \odot \mathcal{H})$ is an $m$-polar fuzzy hyperideal of $H$. In a similar way we can prove that $\widehat{M} \cup(\widehat{M} \odot \widehat{M})$ is an $m$-polar fuzzy hyperideal.

Proposition 3.5. Let $H$ be a regular LA-semihypergroup with a pure left identity e. If $\widehat{M}$ is an m-polar fuzzy right hyperideal of $H$, then $\widehat{M}(x * y)=\widehat{M}(y * x)$ holds for all $x, y \in H$.

Proof. Let $\widehat{M}$ be an $m$-polar fuzzy right hyperideal of a regular LA-semihypergroup $H$ with a pure left identity $e$. Let $x, y \in H$. Since $H$ is regular, $x \in(x * a) * x$ and $y \in(y * b) * y$ for some $a, b \in H$. Now by using the medial and paramedial laws, we get

$$
\begin{aligned}
x * y & \subseteq((x * a) * x) *((y * b) * y) \\
& =(y * x) *((y * b) *(x * a)) .
\end{aligned}
$$


Since $\widehat{M}$ is an $m$-polar fuzzy right hyperideal, then for every $w \in x * y \subseteq(y * x) *((y * b) *(x * a))$, we have

$$
\begin{aligned}
\inf _{w \in x * y}\{\widehat{M}(w)\} & \succeq \inf _{w_{1} \in(y * x) *((y * b) *(x * a))}\left\{\widehat{M}\left(w_{1}\right)\right\} \\
& \succeq \inf _{s \in y * x}\{\widehat{M}(s)\}
\end{aligned}
$$

Again by using the medial and paramedial laws, we get

$$
\begin{aligned}
y * x & \subseteq((y * b) * y) *((x * a) * x) \\
& =(x * y) *((x * a) *(y * b)) .
\end{aligned}
$$

Since $\widehat{M}$ is an $m$-polar fuzzy right hyperideal, for every $t \in y * x \subseteq(x * y) *((x * a) *(y * b))$, we have

$$
\begin{aligned}
\inf _{t \in y * x}\{\widehat{M}(t)\} & \succeq \inf _{t_{1} \in(x * y) *((x * a) *(y * b))}\left\{\widehat{M}\left(t_{1}\right)\right\} \\
& \succeq \inf _{p \in x * y}\{\widehat{M}(p)\},
\end{aligned}
$$

This shows that $\widehat{M}(x * y)=\widehat{M}(y * x)$ holds for all $x, y \in H$.

Lemma 3.3. If $H$ is a left regular LA-semihypergroup, then every m-polar fuzzy left (resp., right) hyperideal $\widehat{M}$ of $H$ is an m-polar fuzzy idempotent.

Proof. Let $\widehat{M}$ be any $m$-polar fuzzy left hyperideal of a left regular LA-semihypergroup $H$ with a pure left identity. Then $\widehat{M} \odot \widehat{M} \subseteq \mathcal{H} * \widehat{M} \subseteq \widehat{M}$. Since $H$ is left regular, for every $a \in H$, there exists $x \in H$ such that $a \in x *(a * a)$. By Lemma 2.1, we have $a \in x *(a * a)=a *(x * a)$. Therefore

$$
\begin{aligned}
(\widehat{M} \odot \widehat{M})(a) & =\sup _{a \in a *(x * a)}\left\{\min \left\{\widehat{M}(a), \inf _{s \in x * a} \widehat{M}(s)\right\}\right\} \\
& \succeq \min \{\widehat{M}(a), \widehat{M}(a)\} \\
& =\widehat{M}(a) .
\end{aligned}
$$

Thus we get $\widehat{M} \subseteq \widehat{M} \odot \widehat{M}$. This implies that $\widehat{M} \odot \widehat{M}=\widehat{M}$. Hence this shows that $\widehat{M}$ is $m$-polar fuzzy idempotent. The other case can be proved in a similar way.

\section{Conclusions}

In this paper, we studied the hyperideal-structure of $m$-polar fuzzy sets in left almost semihypergroups. We defined $m$-polar fuzzy sub LA-semihypergroups and $m$-polar fuzzy left (right, two sided) hyperideal in a left almost semihypergroup and used these $m$-polar fuzzy left (right, two sided) hyperideals to characterize some classes of left almost semihypergroups.

Acknowledgements. The authors would like to thank Deanship of Scientific Research at Majmaah University for supporting this work under Project Number 35/37. 


\section{REFERENCES}

[1] F. Marty, Sur une generalization de la notion de group, 8th Congres Math. Scandinaves, Stockholm, (1934), 45-49.

[2] P. Corsini, Prolegomena of hypergroup theory, Second edition, Aviani editor, (1993).

[3] T. Vougiouklis, Hyperstructures and their representations, Hadronic Press, Florida, (1994).

[4] B. Davvaz, Semihypergroup Theory. Elsevier /Academic Press, London, 2016.

[5] K. Hila and J. Dine, On hyperideals in left almost semihypergroups, ISRN Algebra, 2011 (2011), Article ID 953124.

[6] N. Yaqoob, P. Corsini and F. Yousafzai, On intra-regular left almost semihypergroups with pure left identity, J. Math. 2013 (2013), Art. ID 510790.

[7] F. Yousafzai, P. Corsini, Some charactrization problems in LA-semihypergroups, J. Algebra Number Theory, Adv. Appl. 10(1-2) (2013), 41-55.

[8] V. Amjad, K. Hila, F. Yousafzai, Generalized hyperideals in locally associative left almost semihypergroups, New York J. Math. 20 (2014), 1063-1076.

[9] F. Yousafzai, K. Hila, P. Corsini, A. Zeb, Existence of non-associative algebraic hyper-structures and related problems, Afr. Mat. 26(5) (2015), 981-995.

[10] L. A. Zadeh, Fuzzy Sets, Inform. Control, 8 (1965), 338-353.

[11] A. Rosenfeld, Fuzzy groups, J. Math. Anal. Appl. 35 (1971), 512-517.

[12] J. M. Anthony and H. Sherwood, Fuzzy subgroups redefined, J. Math. Anal. Appl., 69 (1979), 124-130.

[13] H. Sherwood, Products of fuzzy subgroups, Fuzzy Sets Syst. 11 (1983), 79-89.

[14] M. Asaad, Groups and fuzzy subgroups, Fuzzy Sets Syst. 39 (1991), 323-328.

[15] N. P. Mukherjee and P. Bhattacharya, Fuzzy normal subgroups and fuzzy cosets, Inform. Sci. 34 (1984), 225-239.

[16] P. S. Das, Fuzzy groups and level subgroups, J. Math. Anal. Appl. 84 (1981), 264-269.

[17] B. Davvaz, Fuzzy hyperideals in semihypergroups, Italian J. Pure Appl. Math. 8 (2000), 67-74.

[18] B. Davvaz, I. Cristea, Fuzzy algebraic hyperstructures. An introduction. Studies in Fuzziness and Soft Computing, Springer, (2015).

[19] W. R. Zhang, Bipolar fuzzy sets, Proc. FUZZ-IEEE, (1998), 835-840.

[20] J. Chen, S.-G. Li, S. Ma and X. W, m-polar fuzzy sets: An extension of $m$-polar fuzzy sets, Sci. World J. 2014 (2014), Art. ID 416530 .

[21] M. Akram and A. Farooq, m-polar fuzzy Lie ideals of Lie algebras, Quasigroups Relat. Syst. 24(2) (2016), 141-150.

[22] M. Akram, A. Farooq and K. P. Shum, On m-polar fuzzy Lie subalgebras, Italian J. Pure Appl. Math. 36 (2016) 445-454

[23] M. Akram and N. Waseem, Certain metrics in m-polar fuzzy graphs, New Math. Nat. Comput. 12(2) (2016), 135-155.

[24] M. Akram and H. R. Younas, Certain types of irregular m-polar fuzzy graphs, J. Appl. Math. Comput. 53(1-2) (2017), 365-382.

[25] A. Farooq, G. Ali and M. Akram, On m-polar fuzzy groups, Int. J. Algebra Stat. 5(2) (2016), 115-127.

[26] M. Sarwar and M. Akram, Novel applications of $m$-polar fuzzy concept lattice, New Math. Nat. Comput. 13(3) (2017), 261-287.

[27] M. Sarwar and M. Akram, New applications of m-polar fuzzy matroids, Symmetry, 9(12) (2017), Art. ID 319.

[28] M. Akram, m-polar fuzzy graphs, Studies in Fuzziness and Soft Computing, vol 371, Springer, (2019).

[29] A. Al-Masarwah and A. G. Ahmad, $m$-polar $(\alpha, \beta)$-fuzzy ideals in BCK/BCI-algebras, Symmetry, 11 (2019), Art. ID 44. 